\title{
A set of isogenic auxotrophic strains for constructing multiple gene deletion mutants and parasexual crossings in Aspergillus niger
}

\author{
Jing Niu ${ }^{1} \cdot$ Mark Arentshorst $^{1} \cdot$ Felix Seelinger $^{1} \cdot$ Arthur F. J. Ram $^{1}$ • \\ Jean Paul Ouedraogo ${ }^{1,2}$
}

Received: 21 November 2015 / Revised: 27 April 2016 / Accepted: 5 May 2016 / Published online: 1 June 2016

(C) The Author(s) 2016. This article is published with open access at Springerlink.com

\begin{abstract}
To construct a set of isogenic auxotrophic strains in Aspergillus niger suited for creating multiple gene deletion mutants and executing parasexual crossings, we have combined mutations in genes involved in colour pigmentation ( fwnA and olvA) with well-selectable auxotrophic markers (pyrG, nicB, $\arg B$, and adeA). All markers, except for the $\operatorname{pyr} G$ marker, were introduced by targeted deletion, omitting UV mutagenesis of the strains. Aspergillus ory$z a e$ orthologous genes of the $\arg B$, nicB, and adeA markers were used as heterologous selection markers, and all markers were shown to complement to respective auxotrophic A. niger mutants. A quadruple auxotrophic marker was further constructed suitable for multiple gene deletions. Genome sequencing of two auxotrophic colour mutants JN3.2 (olvA::pyrG, $\arg B:: h y g B)$ and JN6.2 (olvA::pyrG, nic $B::$ hyg $B$ ) revealed four SNPs between them in non-coding regions, indicating a high level of isogenicity between both strains. The availability of near-isogenic complementary auxotrophic colour mutants facilitates the selection of diploids and the isolation of haploid segregants from the diploid using the parasexual cycle.
\end{abstract}

Communicated by Olaf Kniemeyer.

Electronic supplementary material The online version of this article (doi:10.1007/s00203-016-1240-6) contains supplementary material, which is available to authorized users.

Arthur F. J. Ram

a.f.j.ram@biology.leidenuniv.nl

1 Molecular Microbiology and Biotechnology, Institute of Biology Leiden, Leiden University, Sylviusweg 72, 2333 BE Leiden, The Netherlands

2 Present Address: Centre for Structural and Functional Genomics, Concordia University, 7141 Sherbrooke St. W., Montreal, QC H4B 1R6, Canada
Keywords Isogenic strains · Auxotrophy $\cdot$ Multiple markers $\cdot$ Parasexual crossing

\section{Introduction}

Aspergillus niger has attracted considerable interest as cell factories for the production of organic compounds (citric acid and secondary metabolites) or (recombinant) proteins (Andersen et al. 2013; Meyer et al. 2015; Pel et al. 2007; Ward 2012). A. niger is not only an important cell factory, it also has become an important model system for fungal development (Krijgsheld et al. 2013; Wösten et al. 2013). System biology-based approaches in combination with targeted metabolic engineering techniques are important tools to study and optimize production processes (Caspeta and Nielsen 2013; Jacobs et al. 2009). With relative ease, gene knockouts can be made using the ku70 mutants (Carvalho et al. 2010; Meyer et al. 2007) in combination with split marker approaches (Nielsen et al. 2006; Goswami 2012; Arentshorst et al. 2015a). Together with tools for controlled overexpression of genes using the tetracycline promoter system (Meyer et al. 2011), metabolic engineering can be efficiently performed. A limiting factor for metabolic engineering in $A$. niger is the limited number of isogenic auxotrophic mutants with multiple auxotrophic markers, in which multiple gene deletion mutants can be made quickly without the need to recycle the selection markers. Selection markers such as the $p y r G$ marker or the amdS marker are counter-selectable, but when multiple deletions need to be made, these markers need to be recycled, which is time-consuming. To overcome this limitation, we have selected the $n i c B$ gene (encoding nicotinate mononucleotide pyrophosphorylase; Verdoes et al. 1994), the $\arg B$ gene (encoding ornithine carbamoyltransferase; Lenouvel et al. 
2002), and the adeA gene (encoding phosphoribosylaminoimidazole-succinocarboxamidesynthase) (Jin et al. 2004; Ugolini and Bruschi 1996) of A. niger to construct nearisogenic auxotrophic marker strains containing four auxotrophic markers (pyrG, nicB, adeA, and $\arg B$ ). In combination with dominant selection markers such as hygromycin resistance (Punt and van den Hondel 1992), phleomycin resistance (Punt and van den Hondel 1992), and AmdS selection (Kelly and Hynes 1985), seven different markers are available for strain construction.

The lack of a sexual cycle in A. niger limits easy crossing of two strains to combine interesting properties or to construct double mutants. Despite the lack of a sexual cycle, the parasexual cycle can be used to combine genetic traits in A. niger (Pontecorvo et al. 1953; Swart et al. 2001). The parasexual cycle includes the selection of a heterokaryon and subsequently the selection of a diploid strain. The frequency by which diploids are formed from a heterokaryotic mycelium in A. niger is very low, and selection of diploids can be accomplished by crossing strains that have complementary auxotrophic and complementary spore colour markers. Only when a diploid is formed, the resulting colony will produce solely black conidiospores which can be easily detected by eye. The genes encoding proteins involved in spore melanin production in A. niger have been identified (Jørgensen et al. 2011). Several studies, mainly conducted by Bos et al., have reported on the isolation of A. niger colour and auxotrophic mutants [see for review (Swart et al. 2001)]. However, most of these mutants were isolated by UV treatment. Although carried out with caution and relative high survival rates, unwanted random mutations are inevitable, leading to possible growth defects. By targeted deletion of spore colour genes and auxotrophies, we constructed a set of near-isogenic strains suitable for parasexual crossings (Niu et al. 2016). We performed genome sequencing of two auxotrophic colour mutants and confirmed the near-isogenicity between these auxotrophic mutants.

\section{Materials and methods}

\section{Strains and growth conditions}

The A. niger strains used in this study are listed in Table 1 . Auxotrophic strains are deposited at the Fungal Genetic Stock Centre. A. niger strains were grown on minimal medium (MM) (Bennet and Lasure 1991) or on complete medium $(\mathrm{CM})$ consisting of minimal medium with the addition of $5 \mathrm{~g} / \mathrm{L}$ yeast extract and $1 \mathrm{~g} / \mathrm{L}$ casamino acids. When required, $10 \mathrm{mM}$ uridine, $200 \mu \mathrm{g} / \mathrm{mL}$ L-arginine, $2.5 \mu \mathrm{g} / \mathrm{mL}$ nicotinamide, $100 \mu \mathrm{g} / \mathrm{mL}$ hygromycin, or $40 \mu \mathrm{g} / \mathrm{mL}$ phleomycin was added. Adenine was directly added from the solid stock to the medium to a final concentration of $200 \mathrm{mg} / \mathrm{L}$ after autoclaving and dissolved by mixing. Fluoroacetamide (FAA) and 5-fluoroorotic acid (5-FOA) counter-selection was performed as described (Carvalho et al. 2010; Arentshorst et al. 2012) to remove the amdS marker and the $p y r G$ marker, respectively.

\section{Molecular biological techniques}

Transformation of $A$. niger and chromosomal DNA isolation of A. niger and Aspergillus oryzae were performed
Table 1 Strains used in this study

\begin{tabular}{|c|c|c|}
\hline Name & Genotype/description & Reference/source \\
\hline $\mathrm{N} 402$ & $\operatorname{csp} A 1$, derivative of $\mathrm{N} 400$ & Bos et al. (1988) \\
\hline A. oryzae & ATCC16868 & - \\
\hline MA169.4 & kusA::amdS, pyrG $G^{-}$ & Carvalho et al. (2010) \\
\hline MA100.1 & 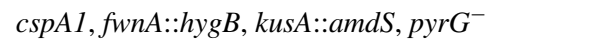 & Jørgensen et al. (2011) \\
\hline AW8.4 & cspA1, olvA::AopyrG in MA169.4 & Jørgensen et al. (2011) \\
\hline JN3.2 & $\arg B:: h y g B$, olvA::AopyrG (derived from AW8.4) & This study \\
\hline JN6.2 & nicB::hygB, olvA::AopyrG (derived from AW8.4) & This study \\
\hline JN1.17.1 & $\arg B:: h y g B$ in MA169.4 & This study \\
\hline OJP3.1 & nicB::phleo in MA169.4 & This study \\
\hline OJP1.1 & adeA::pyrG in MA169.4 & This study \\
\hline MA322.2 & ku70::amdS, nicB::AopyrG in MA169.4 & This study \\
\hline MA323.1 & ku70::amdS, $\Delta$ nic $B^{-}$, pyrG $G^{-}$ & This study \\
\hline MA328.2 & ku70::amdS, $\triangle$ nic $B^{-}$, adeA::AopyrG & This study \\
\hline MA329.1 & ku70::amdS, $\Delta$ nic $B^{-}, \Delta a d e A^{-}, p y r G^{-}$ & This study \\
\hline MA334.2 & ku70::amdS, $\Delta$ nic $B^{-}, \Delta$ ade $A^{-}, \arg B:: A o p y r G$ & This study \\
\hline MA335.3 & ku70::amdS, $\Delta$ nicB $B^{-}, \Delta a d e A^{-}, \Delta \arg B^{-}, p y r G^{-}$ & This study \\
\hline
\end{tabular}


according to (Meyer et al. 2010). Southern blot analysis was performed according to (Sambrook and Russell 2001). $\alpha-{ }^{32} \mathrm{P}-\mathrm{dCTP}$-labelled probes were synthesized using the Rediprime II kit (Amersham, GE Healthcare), according to the instructions of the manufacturer. Restriction and ligation enzymes were obtained from Thermo Scientific and used according to the instructions of the manufacturer. PCR was performed with Phire Hot Start II DNA polymerase or Phusion DNA polymerase (Thermo Scientific). Sequencing was performed by Macrogen.

\section{Construction of plasmids and deletion cassettes}

The deletion cassettes for the $\arg B, \operatorname{nic} B$, and $a d e A$ genes of $A$. niger were constructed with the hygB, phleo, and pyrG selection markers, respectively. The plasmid used to disrupt the $\arg B$ gene (An14g03400) with the hygromycin selection marker was constructed as follows: $\sim 0.8-\mathrm{kb}$ DNA fragments flanking the $\arg B$ ORF were amplified by PCR using N402 genomic DNA as template, with primers listed in Supplementary Table 1. The PCR products were cloned into pJet1.2 (Thermo Scientific). The $5^{\prime}$ flank of $\arg B$ was excised from pJet1.2 using KpnI/HindIII and inserted into the same site of pBlueScript II Sk(+) to obtain plasmid pJN3.3. Subsequently, pJN3.3 was digested with HindIII/NotI and used in a three-way ligation with the $3^{\prime}$ flank of $\arg B$ excised from pJet1.2 using XhoI/NotI and the 3-kb HindIII/XhoI fragment containing the $h y g B$ gene, obtained from plasmid p $\Delta 2380$ (Damveld et al. 2008), resulting in the $\arg B$ disruption plasmid pJN4.5. The $\arg B$ gene deletion cassette was amplified by PCR using pJN4.5 DNA as template with primers $\operatorname{argBKO} 1$ and $\operatorname{argBKO} 4$ and the purified linear PCR fragment was used for subsequent transformation to A. niger strain MA169.4 $\left(\mathrm{ku}^{2} 0^{-}\right.$, pyrG $\left.\mathrm{G}^{-}\right)$ to give JN1.17.1 (ku70-, pyrG $\left.{ }^{-}, \Delta \arg B:: h y g B\right)$ or to $A$. niger strain AW8.4 (ku70-, $\Delta$ olvA::AOpyrG), resulting in

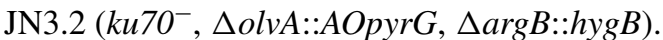

The same approach was used to construct the disruption cassettes of the nicB gene (An11g10910) of A. niger with either the phleomycin or hygromycin marker. The DNA fragments flanking the nicB ORF were amplified from N402 genomic DNA, with primers listed in Supplementary Table 1. After cloning in pJet1.2, the 5 'flank of $n i c B$ was isolated as a $K p n \mathrm{I} / \mathrm{XhoI}$ fragment and inserted

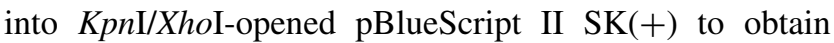
plasmid pJN8.1. Subsequently, the 1.9-kb XhoI-HindIII fragment containing phleo expression cassette, obtained from plasmid pMA299, or the 3.1-kb XhoI-HindIII fragment containing $h y g B$ expression cassette, obtained from plasmid p $\Delta 2380$ (Damveld et al. 2008), together with the HindIII/NotI isolated $3^{\prime}$ flank of nicB, were ligated into XhoI/NotI-opened pJN8.1, resulting in the nicB::phleo disruption plasmid $\mathrm{pJN} 10.1$ or nicB::hygB disruption plasmid pJN9.1. The nicB gene deletion cassettes were amplified by PCR using pJN10.1 or pJN9.1 as template with primer NicBKO1 and NicBKO4 and used for transformation to A. niger strain MA169.4 $\left(\mathrm{ku}^{-} \mathrm{O}^{-}, \mathrm{pyr} \mathrm{G}^{-}\right)$to give OJP3.1 (ku70-, pyrG ${ }^{-}, \Delta$ nicB::phleo $)$ or to A. niger strain AW8.4 (ku70-, $\Delta$ olvA::AOpyrG), resulting in JN6.2 $\left(\right.$ ku $70^{-}, \Delta$ olvA::AOpyrG, $\triangle$ nicB::hygB $)$.

To construct the disruption cassette of adeA gene (An11g10150), the flanking regions of the gene were amplified by PCR from N402 genomic DNA with primers Fw_adeA_5' and Rev_adeA_5' to obtain the 0.9-kb 5'flanking region and Fw_adeA_3'and Rev_adeA_3' to obtain the $0.7-\mathrm{kb} 3^{\prime}$ flanking region (Supplementary Table 1). The 1.8-kb A. nidulans pyrG selection marker was amplified by PCR from the plasmid pCRpyrGAN (Ouedraogo et al. 2015) with the primers Fw_pyrG_adeA and Rev_pyrG_adeA which contain complementary sequence of Rev_adeA-5' and Fw_adeA-3', respectively (Supplementary Table 1). The adeA::Anid_pyrG deletion cassette was obtained by a fusion PCR of the three purified PCR products, followed by cloning of the 3.4-kb fusion PCR product into pJet1.2, resulting in plasmid pOJP1 and used for transformation to A. niger strain MA169.4 $\left(k u 70^{-}, p y r G^{-}\right)$ to give OJP1.1 ( $\left.k u 70^{-}, \triangle a d e A:: p y r G\right)$. Proper deletion of the $n i c B$, ade $A$, and $\arg B$ genes was confirmed by Southern blot analysis (Supplementary Figures. 1-3).

For complementation studies, $\arg B$, nicB, and adeA genes, including their promoter and terminator regions, were amplified from wild-type A. oryzae and A. niger genomic DNA with appropriate primer pairs described in the Supplementary Table 1. The respective complementing gene fragments were cloned into pJet1.2 (Thermo Scientific) and sequenced (Table 2). The plasmids pOJP5 (pJet1.2_Anig.argB), pOJP4 (pJet1.2_Anig.nicB), pOJP3 (pJet1.2_Anig.adeA), pJN29 (pJet1.2_Aory.argB), pJN30 (pJet1.2_Aory.nicB), and pJN31 (pJet1.2_Aory.adeA) were used to complement the respective auxotrophic mutants.

\section{Recyclable split marker strategy for creation of a strain with multiple auxotrophies}

To construct an A. niger strain with multiple auxotrophies, it was necessary to use a recyclable split marker approach. Therefore, auxotrophic marker-specific direct repeats (DR) surrounding the $A O p y r G$ selection marker were introduced by PCR. By selecting on 5-FOA, the AOpyrG marker was removed. The recyclable split marker approach is outlined in Fig. 1; see Supplementary Table 1 for primer sequences. Strain MA169.4 $\left(k u 70^{-}\right.$, pyrG $\left.G^{-}\right)$was used as starting strain to first delete the $n i c B$ gene and, subsequently, adeA and the 
Table 2 Plasmids used in this study

\begin{tabular}{lll}
\hline Name & Description & Reference/source \\
\hline pJN3.3 & 5'flank of $\operatorname{argB}$ in pBluescript II SK(+) & This study \\
p $\Delta 2380$ & $\Delta$ ugmB::hygB deletion cassette & Damveld et al. (2008) \\
pJN4.5 & pBluescript_argB::hygB & This study \\
pJN8.1 & 5'flank of nicB in pBluescript II SK(+) & This study \\
pMA299 & pBluescript_phleo & This study \\
pJN10.1 & pBluescript_nicB::phleo & This study \\
pCRpyrGAN & Containing the full gene of A. nidulans pyrG & Ouedraogo et al. (2015) \\
pOJP1 & pJet1.2_adeA::pyrG & This study \\
pOJP5 & pJet1.2_Anig.argB & This study \\
pOJP4 & pJet1.2_Anig.nicB & This study \\
pOJP3 & pJet1.2_Anig.adeA & This study \\
pJN29 & pJet1.2_Aory.argB & This study \\
pJN30 & pJet1.2_Aory.nicB & This study \\
pJN31 & pJet1.2_Aory.adeA & This study \\
pAO4-13 & Containing full pyrG gene of A. oryzae & de Ruiter-Jacobs et al. (1989) \\
\hline
\end{tabular}

WT

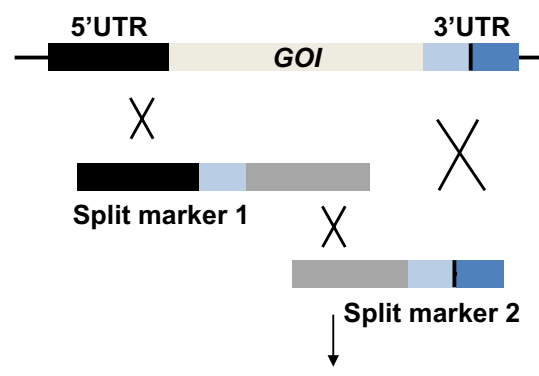

$\Delta G O I$, pyrG ${ }^{+}$

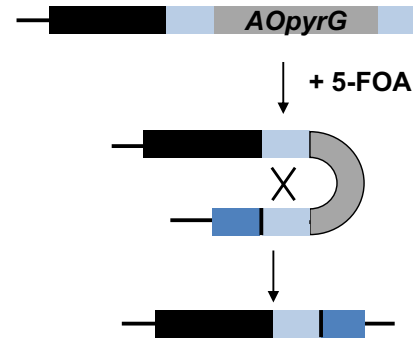

Fig. 1 Schematic representation of the recyclable split marker approach for multiple gene deletion mutants. Deletion of the gene of interest (GOI) by split marker approach with recycling of the Aspergillus oryzae pyrG marker. The split marker fragments 1 and 2 are used during transformation to knock out the GOI by homologous recombination which generates a uridine prototroph $\left(\right.$ pyrG $\left.G^{+}\right)$strain. The pyr $G$ marker is subsequently looped out by 5-FOA selection, and the resulting $\mathrm{pyrG}^{-}$strain is suitable for a second gene deletion with the pyrG marker. The split marker approach is described previously (Arentshorst et al. 2015a)

$\arg B$ marker. All strains containing single, double, triple, and the quadruple auxotrophic strain are listed in Table 1. Correct integration of split marker fragments and successful loop out of the $A O p y r G$ was confirmed by Southern blot analysis for all strains and shown for MA335.3 in Supplementary Figures. 1-3).

\section{A. niger parasexual cycle}

Heterokaryon formation and selection for diploids was performed as described (Pontecorvo et al. 1953). Segregation of diploids by benomyl was performed essentially as described (Bos et al. 1988) with slight modifications (Niu et al. 2016).

\section{Sequencing and analysis}

Genome sequencing of JN3.2 (olvA::pyrG, $\arg B:: h y g B$ ) and JN6.2 (olvA::pyrG, nicB::hygB) was performed using NGS platform (Illumina GA) as described (Park et al. 2014). Sequencing was performed at ServiceXS, Leiden, The Netherlands. SNPs between JN3.2 and JN6.2 were identified using A. niger strain ATCC1015 (http://genome. jgi-psf.org/pages/search-for-genes.jsf?organism=Aspni5) as reference genome. For each SNP, it was verified whether the SNP was in a predicted protein-encoding region using the A. niger 3.0 genome at JGI using the SNP coordinates (Park et al. 2014).

\section{Results and discussion}

\section{Construction and characterization of $\arg B, \operatorname{nic} B$, and ade $A$ auxotrophic mutants}

Deletion constructs nicB::hygB, $\arg B:: p h l e o$, and adeA::pyrG were transformed to strain MA169.4 $\left(k u 70^{-}\right.$, pyr $G^{-}$), and hygromycin, phleomycin resistant, or uridine prototrophic transformants were obtained and purified. Proper deletion of the respective markers was verified by diagnostic PCRs (data not shown) and by testing the growth 
Fig. 2 Supplementation test of the auxotrophic $A$. niger mutants. $10 \mu \mathrm{L}$ of a spore stock $\left(1 \times 10^{7}\right.$ conidia $\left./ \mathrm{mL}\right)$ of each auxotrophic strain and the parental strain (MA169.4) was inoculated on an MM plate without and with serial concentrations of the respective supplement and incubated at $30{ }^{\circ} \mathrm{C}$ for 3 days for arginine and nicotinamide supplementation test and for 4 days for adenine supplementation test

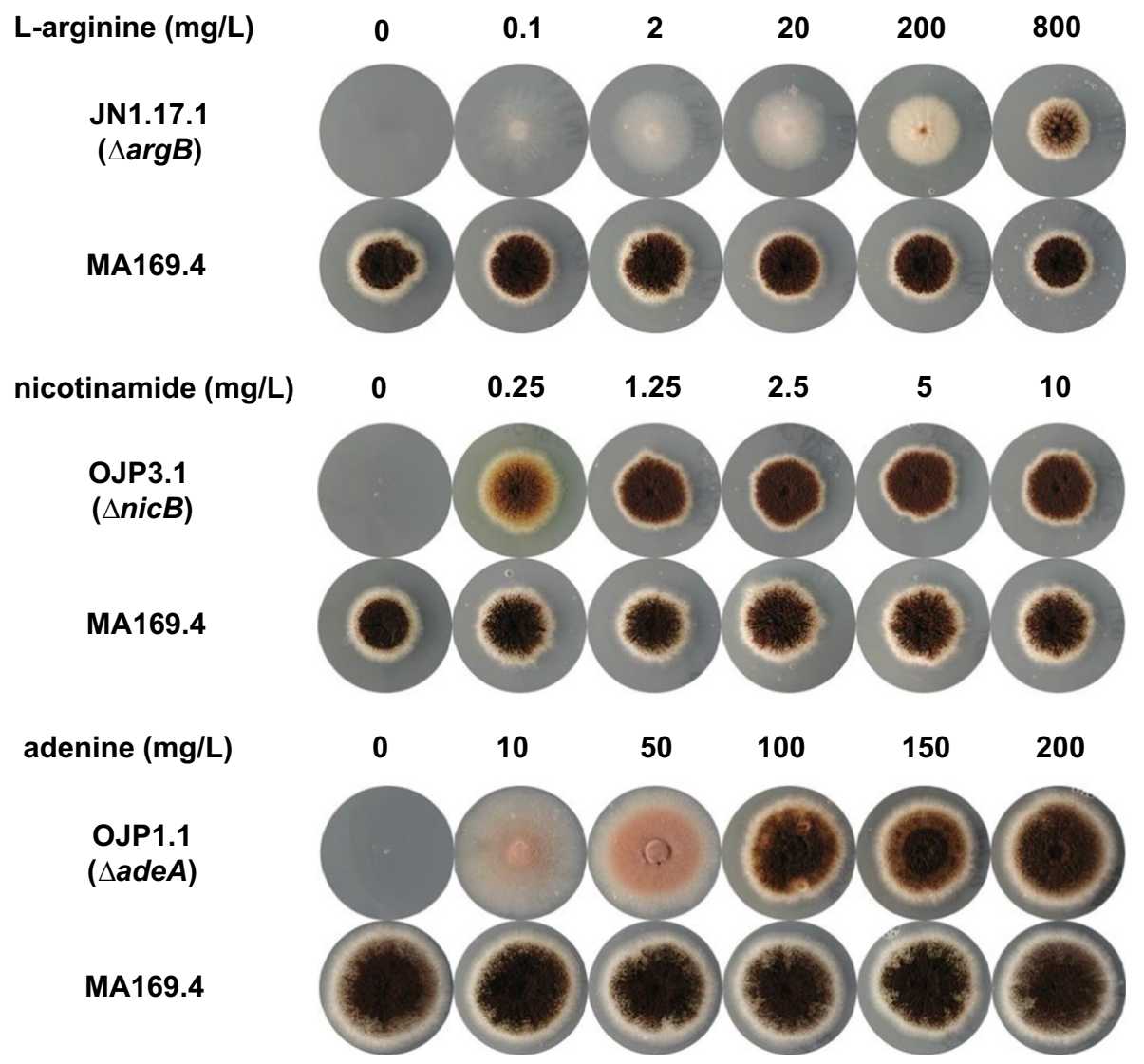

and in materials and methods. This approach allows iterative construction of gene knockouts in A. niger by subsequent recycling of the pyrG marker using counter-selection on 5-FOA, due to the presence of the direct repeated sequences flanking the selection marker. The proper deletion and absence of ectopic copies of the deletion cassettes in the quadruple auxotrophic strain MA335.3 was confirmed by Southern blot analysis (Supplemental Figures. 1-3) and characterized by the inability to growth in the absence of arginine, nicotinamide, adenine, or uridine (Fig. 3). This quadruple auxotrophic strain offers the possibility to delete multiple genes without the need to recycle the selection marker.

\section{The nicB, $\arg B$, and adeA genes from $A$. oryzae are suitable markers for $A$. niger transformation}

To prove that auxotrophic mutants can be complemented by heterologous and homologous markers, DNA fragments containing the $\arg B$, the nicB, and the adeA genes from $A$. oryzae and $A$. niger, including their promoters and $3^{\prime}$ untranslated sequences, were used for the complementation of the respective $A$. niger auxotrophic mutants. Protoplasts of JN1.17.1 ( $\triangle \arg B:: h y g B)$, OJP3.1 ( $\Delta$ nicB::phleo), and OJP1.1 ( $\triangle a d e A:: p y r G)$ were transformed with plasmids containing the corresponding marker genes from

We have constructed a quadruple auxotrophic strain based on the recyclable split marker approach described in Fig. 1 


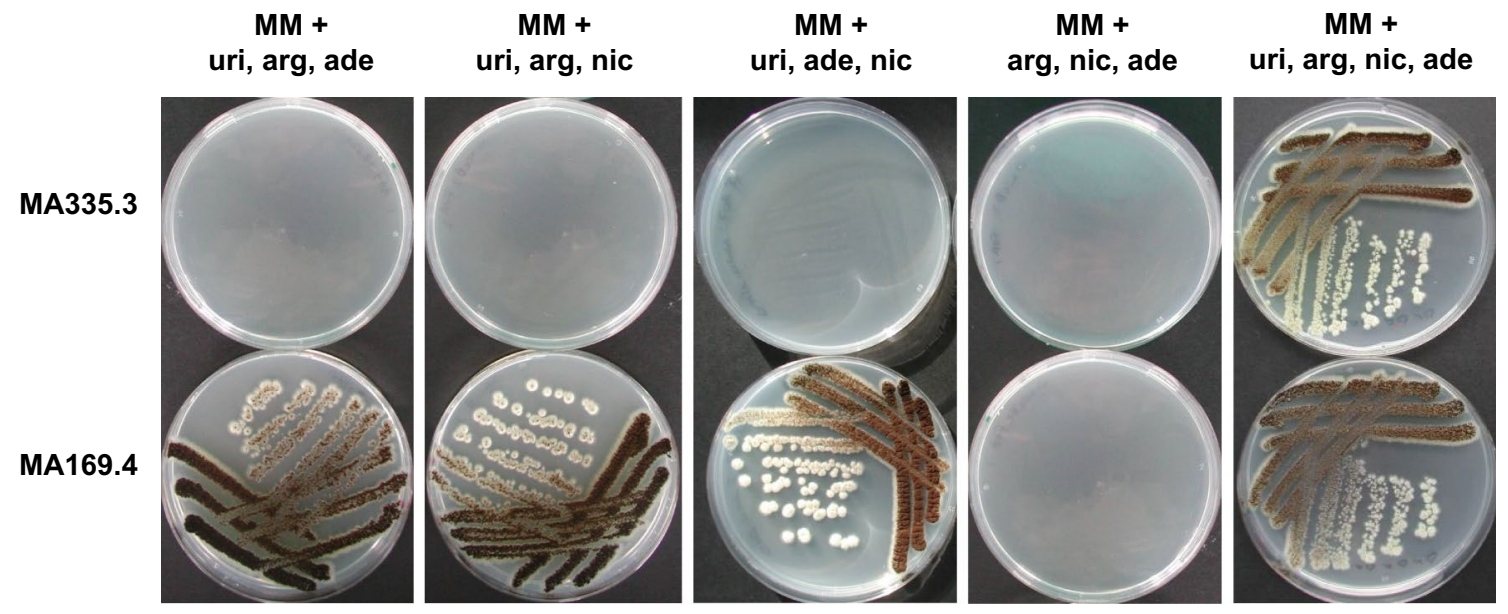

Fig. 3 Growth analysis of the quadruple auxotrophic $A$. niger strain. MA335.3 ( $\triangle$ nicB, $\triangle \arg B, \triangle a d e A, p y r G^{-}$) was plated on solid MM with and without the different supplements at $30^{\circ} \mathrm{C}$, and growth was

A. oryzae or A. niger. Transformants were obtained for the $A$. oryzae heterologous markers, which demonstrated that $n i c B, \arg B$, and adeA of $A$. oryzae complemented the auxotrophy and therefore are suitable markers for $A$. niger transformations. As expected, also all A. niger genes $(\arg B$, $n i c B$, and adeA) were able to complement the respective auxotrophic $A$. niger mutants. The obtained transformants were further analysed to determine whether the A. oryzae marker also complemented the auxotrophies. As shown in Fig. 4, all heterologous genes complement similarly to the homologous A. niger genes. A heterologous marker for gene disruption experiments is preferred as it reduces the homologous integration of the marker gene in the disruption cassette at the homologous site. We have compared the DNA sequence of the different genes markers of A. niger to those of $A$. oryzae by BLASTN (http://blast.ncbi.nlm. nih.gov/) using standard settings. The identity of the coding regions between the different gene markers was 73.3, 72.0 , and $77.8 \%$ for $\arg B$, nicB , and adeA genes, respectively. These values are comparable to the value obtained when comparing the $p y r G$ genes markers of both Aspergillus species. The pyrG gene of $A$. oryzae is identical to the pyrG gene of $A$. niger at $78.6 \%$ and has been so far successfully used to transform A. niger and vice versa (Carvalho et al. 2010; Mattern et al. 1987). It should be noted that complementation analysis in the $\Delta k u 70$ background is not efficient because of the low frequencies of ectopic integration the complementing fragment. To circumvent this limitation, we constructed a curable $k u 70$ deletion strategy (Carvalho et al. 2010). The presence of $k u 70$ repeats around the AmdS selection marker used to disrupt the $k u 70$ gene allows efficient loop out of the AmdS marker via fluoroacetamide counter-selection as described (Arentshorst et al. 2012). An alternative method for easy complementation, analysed after 3 days. The parental strain MA169.4 was taking along the analysis for comparison

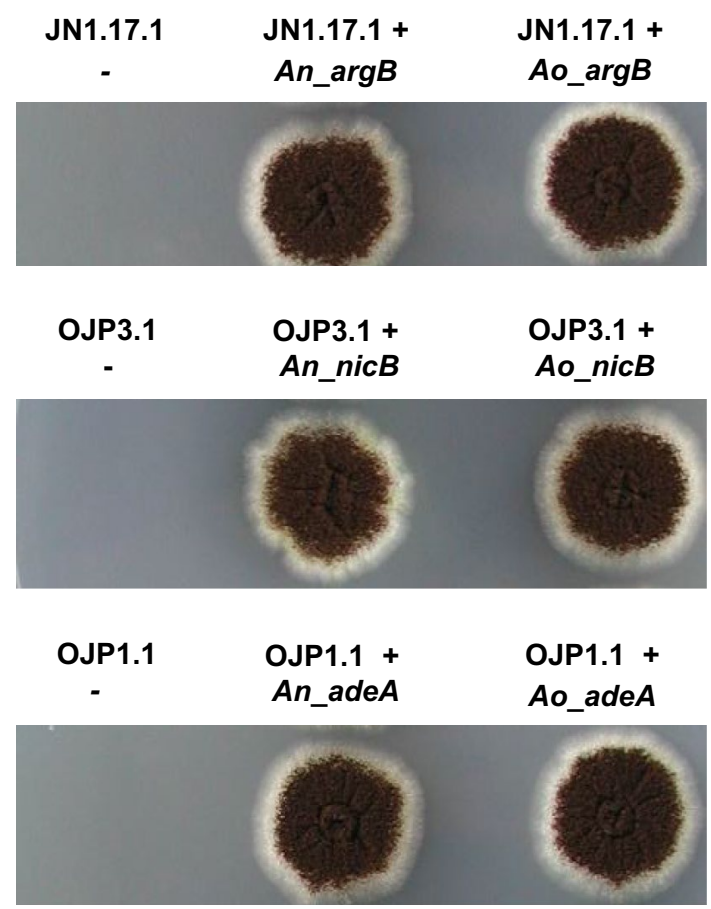

Fig. 4 Growth analysis of the complemented transformants. Spores of JN1.17.1 ( $\left.\triangle \operatorname{argB}, p y r G^{-}\right)$OJP3.1 ( $\Delta$ nicB, pyrG $\left.{ }^{-}\right)$and OJP1.1 $(\triangle a d e A)$ and complemented strains were spotted on selective medium to test complementation of the $\arg B$, nicB, and adeA, respectively, from A. niger (An) or A. oryzae (Ao). Pictures were taken after 3 days of growth at $30^{\circ} \mathrm{C}$

which omits the need for curing the $k u 70$ locus, is the use of a second auxotrophic marker which can be used to target the complementing gene to this locus. For the pyrG marker, an efficient gene targeting method has recently become available (Arentshorst et al. 2015b) which allows targeted 
integration when the complementing fragment is cloned in the $\operatorname{pyr} G$ targeting vector. For example, one could start with a nic $B^{-}$, pyr $G^{-}$strain and use the nicB selection marker for initial deletion of the gene of interest, followed by a complementation experiment in which the complementing fragment is cloned in the pyrG targeting vector which is that transformed to the deletion strain.

\section{Isogenic auxotrophic colour mutants for parasexual crossing in A. niger}

Combining mutations by crossing strains is a powerful genetic tool for strain construction. In Aspergillus nidulans, this method is well established and used in many studies to construct double mutants (Todd et al. 2007). The lack of a sexual cycle in A. niger has limited the use of crossings to combine mutations. However, the use of the parasexual cycle in A. niger (Pontecorvo et al. 1953) has been used extensively for linkage studies in A. niger and can be used to combine mutations (Bos et al. 1988). Straightforward crossing in A. niger requires complementing auxotrophies to select for a heterokaryotic mycelium and preferably colour makers to select for a diploid strain. The frequency by which $A$. niger forms diploids is generally very low (1 in $10^{6}-10^{7}$ spores), and diploids are not easily detected if wildtype strains are used that produce black conidia. By using complementing colour markers, a diploid can be selected as only this diploid will produce black spores, whereas a heterokaryotic mycelium will produce a mix of heterogeneously coloured spores (Pontecorvo et al. 1953). By combining colour mutants ( $w n n A$ and $o l v A$ ) with complementary auxotrophic markers such as $p y r G, n i c B$, or $\arg B$, heterokaryons and diploids can be easily selected. We constructed several auxotrophic colour mutant strains including MA100.1 (fwnA::hygB, pyrG $G^{-}$), JN3.2 (olvA::pyrG, $\left.\arg B:: h y g B\right)$, and JN6.2 (olvA::pyrG, nicB::hygB) (Table 1). In a recently conducted study, JN3.2 has been used for parasexual crossings to obtain haploid segregants (Niu et al. 2016). With these segregants, a bulk segregant analysis was performed to identify SNPs that are closely linked or responsible for the mutant phenotypes (Niu et al. 2016).

To test the isogenicity between two auxotrophic colour mutants JN3.2 (olvA::pyrG, $\arg B:$ :hygB) and JN6.2 (olvA::pyrG, nicB::hygB), the genomes of these strains were sequenced and compared to the genome of the reference ATCC strain. In total, 155 SNPs were found for JN3.2 and JN6.2, respectively, when compared to the ATCC reference strain (Supplementary Table 2). Two SNPs were found to be specific for JN3.2, and two SNPs were specific for JN6.2. None of them were found in predicted open reading frames (Table 3), demonstrating that JN3.2 and JN6.2 are likely to have no mutation affected its phenotype and that they are near-isogenic.
Table 3 SNP comparison JN6.2 and JN3.2

\begin{tabular}{lrllll}
\hline & Position & Allel ATCC & JN6.2 & JN3.2 & Details mutation \\
\hline chr_1_2 & 726,573 & $\mathrm{~T}$ & $\mathrm{~T}$ & $\mathrm{C}$ & Intergenic \\
chr_3_4 & 45,864 & $\mathrm{~T}$ & $\mathrm{~T}$ & $\mathrm{~A}$ & Intergenic \\
chr_8_2 & $2,725,044$ & $\mathrm{G}$ & $\mathrm{A}$ & $\mathrm{G}$ & Intergenic \\
chr_8_2 & $2,725,045$ & $\mathrm{~T}$ & $\mathrm{~A}$ & $\mathrm{~T}$ & Intergenic \\
\hline
\end{tabular}

In conclusion, new auxotrophic strains carrying targeted deletions in the $\arg B, n i c B$, and adeA genes of $A$. niger were constructed. The orthologous genes $\arg B$, nic $B$, and adeA of $A$. oryzae complemented the arginine, nicotinamide, and adenine auxotrophic mutants similar to the endogenous genes and are therefore suitable selection markers for $A$. niger transformations. The quadruple auxotrophic strain MA335.3 $\left(\arg B^{-}\right.$, nic $B^{-}$, ade $A^{-}$, and pyr $G^{-}$) allows rapid deletion of multiple genes the need to recycle selection markers. The targeted deletion of auxotrophic markers instead of selection of auxotrophic strains after UV mutagenesis significantly reduces the occurrence of mutations as genome sequencing of two auxotrophic mutants (JN3.2 and JN6.2) revealed only four SNP between them.

Acknowledgments Jing Niu was supported by a Grant from the China Scholarship Council.

Open Access This article is distributed under the terms of the Creative Commons Attribution 4.0 International License (http://creativecommons.org/licenses/by/4.0/), which permits unrestricted use, distribution, and reproduction in any medium, provided you give appropriate credit to the original author(s) and the source, provide a link to the Creative Commons license, and indicate if changes were made.

\section{References}

Andersen MR, Nielsen JB, Klitgaard A et al (2013) Accurate prediction of secondary metabolite gene clusters in filamentous fungi. Proc Natl Acad Sci 110:E99-E107. doi:10.1073/ pnas. 1205532110

Arentshorst M, Ram AFJ, Meyer V (2012) Using non-homologous end-joining-deficient strains for functional gene analyses in filamentous fungi. Methods Mol Biol 835:133-150

Arentshorst M, Jing N, Ram AF (2015a) Efficient generation of Aspergillus niger knock out strains by combining NHEJ mutants and a split marker approach. In: van den Berg MA, Maruthachalam K (eds) Genetic transformation systems in fungi, vol 1. Springer International Publishing, Cham, Switzerland, pp 263-272

Arentshorst M, Lagendijk EL, Ram AF (2015b) A new vector for efficient gene targeting to the pyrG locus in Aspergillus niger. Fungal Biol Biotechnol 2:2

Bennet JW, Lasure LL (1991) More gene manipulations in fungi. Academic Press, San Diego

Bos CJ, Debets AJ, Swart K, Huybers A, Kobus G, Slakhorst SM (1988) Genetic analysis and the construction of master strains 
for assignment of genes to six linkage groups in Aspergillus niger. Curr Genet 14:437-443. doi:10.1007/BF00521266

Carvalho ND, Arentshorst M, Jin Kwon M, Meyer V, Ram AF (2010) Expanding the $k u 70$ toolbox for filamentous fungi: establishment of complementation vectors and recipient strains for advanced gene analyses. Appl Microbiol Biotechnol 87:1463-1473. doi:10.1007/s00253-010-2588-1

Caspeta L, Nielsen J (2013) Toward systems metabolic engineering of Aspergillus and Pichia species for the production of chemicals and biofuels. Biotechnol J 8:534-544. doi:10.1002/ biot. 201200345

Damveld RA, Franken A, Arentshorst M, Punt PJ, Klis FM, van den Hondel CA, Ram AF (2008) A novel screening method for cell wall mutants in Aspergillus niger identifies UDP-galactopyranose mutase as an important protein in fungal cell wall biosynthesis. Genetics 178:873-881. doi:10.1534/genetics.107.0731

de Ruiter-Jacobs YM, Broekhuijsen M, Unkles SE, Campbell EI, Kinghorn JR, Contreras R, Pouwels PH, van den Hondel CA (1989) A gene transfer system based on the homologous pyrG gene and efficient expression of bacterial genes in Aspergillus oryzae. Curr Genet 16:159-163

Goswami RS (2012) Targeted gene replacement in fungi using a splitmarker approach. Methods Mol Biol 835:255-269

Jacobs DI, Olsthoorn MM, Maillet I et al (2009) Effective lead selection for improved protein production in Aspergillus niger based on integrated genomics. Fungal Genet Biol 46(Suppl 1):S141S152. doi:10.1016/j.fgb.2008.08.012

Jin FJ, Maruyama J, Juvvadi PR, Arioka M, Kitamoto K (2004) Adenine auxotrophic mutants of Aspergillus oryzae: development of a novel transformation system with triple auxotrophic hosts. Biosci Biotechnol Biochem 68:656-662. doi:10.1271/ bbb.68.656

Jørgensen TR, Park J, Arentshorst M et al (2011) The molecular and genetic basis of conidial pigmentation in Aspergillus niger. Fungal Genet Biol 48:544-553. doi:10.1016/j.fgb.2011.01.005

Kelly JM, Hynes MJ (1985) Transformation of Aspergillus niger by the amdS gene of Aspergillus nidulans. EMBO J 4:475-479

Krijgsheld P, Bleichrodt R, van Veluw GJ, Wang F, Müller WH, Dijksterhuis J, Wösten HA (2013) Development in Aspergillus. Stud Mycol 74:1-29. doi:10.3114/sim0006

Lenouvel F, van de Vondervoort PJ, Visser J (2002) Disruption of the Aspergillus niger $\arg B$ gene: a tool for transformation. Curr Genet 41:425-432. doi:10.1007/s00294-002-0320-0

Mattern IE, Unkles S, Kinghorn JR, Pouwels PH, van den Hondel CA (1987) Transformation of Aspergillus oryzae using the Aspergillus niger pyrG gene. Mol Gen Genet 210:460-461. doi:10.1007/ BF00327197

Meyer V, Arentshorst M, El-Ghezal A, Drews AC, Kooistra R, van den Hondel CA, Ram AF (2007) Highly efficient gene targeting in the Aspergillus niger kusA mutant. J Biotechnol 128:770-775. doi:10.1016/j.jbiotec.2006.12.021

Meyer V, Ram AFJ, Punt PJ (2010) Genetics, genetic manipulation, and approaches to strain improvement of filamentous fungi. In: Baltz RH, Demain AL, Davies JE (eds) Manual of Industrial Microbiology and Biotechnology. ASM Press, Washington, DC, pp 318-329

Meyer V, Wanka F, van Gent J, Arentshorst M, van den Hondel CA, Ram AF (2011) Fungal gene expression on demand: an inducible, tunable, and metabolism-independent expression system for Aspergillus niger. Appl Environ Microbiol 77:2975-2983. doi:10.1128/AEM.02740-10

Meyer V, Fiedler M, Nitsche B, King R (2015) The cell factory Aspergillus enters the big data era: opportunities and challenges for optimising product formation. Adv Biochem Eng Biotechnol 149:91-132. doi:10.1007/10_2014_297

Nielsen ML, Albertsen L, Lettier G, Nielsen JB, Mortensen UH (2006) Efficient PCR-based gene targeting with a recyclable marker for Aspergillus nidulans. Fungal Genet Biol 43:54-64

Niu J, Arentshorst M, Nair PDS, Dai Z, Baker S, Frisvad JC, Nielsen KF, Punt PJ, Ram AFJ (2016) Systems genetics to characterize classical mutants in the industrial host Aspergillus niger: LaeA is required for citric acid production and the production of some secondary metabolites. G3 (Bethesda) 6:193-204. doi:10.1534/ g3.115.024067

Ouedraogo JP, Arentshorst M, Nikolaev I, Barends S, Ram AF (2015) I-SceI-mediated double-strand DNA breaks stimulate efficient gene targeting in the industrial fungus Trichoderma reesei. Appl Microbiol Biotechnol 99:10083-10095. doi:10.1007/ s00253-015-6829-1

Park J, Tefsen B, Arentshorst M, Lagendijk E, van den Hondel CA, van Die I, Ram AFJ (2014) Identification of the UDP-glucose4-epimerase required for galactofuranose biosynthesis and galactose metabolism in A. niger. Fungal Biol Biotechnol 1:6

Pel HJ, de Winde JH, Archer DB et al (2007) Genome sequencing and analysis of the versatile cell factory Aspergillus niger CBS 513.88. Nat Biotechnol 25:221-231. doi:10.1038/nbt1282

Pontecorvo G, Roper JA, Forbes E (1953) Genetic recombination without sexual reproduction in Aspergillus niger. J Gen Microbiol 8:198-210. doi:10.1099/00221287-8-1-198

Punt PJ, van den Hondel CA (1992) Transformation of filamentous fungi based on hygromycin B and phleomycin resistance markers. Methods Enzymol 216:447-457. doi:10.1016/0076-6879(92)16041-H

Sambrook J, Russell DW (2001) Molecular cloning: a laboratory manual, 3rd edn. Cold Spring Harbor Laboratory Press, New York

Swart K, Debets AJ, Bos CJ, Slakhorst M, Holub EF, Hoekstra RF (2001) Genetic analysis in the asexual fungus Aspergillus niger. Acta Biol Hung 52:335-343. doi:10.1556/ABiol.52.2001.2-3.18

Todd RB, Davis MA, Hynes MJ (2007) Genetic manipulation of Aspergillus nidulans: meiotic progeny for genetic analysis and strain construction. Nat Protoc 2:811-821. doi:10.1038/ nprot.2007.112

Ugolini S, Bruschi CV (1996) The red/white colony color assay in the yeast Saccharomyces cerevisiae: epistatic growth advantage of white ade8-18, ade 2 cells over red ade 2 cells. Curr Genet 30:485-492. doi:10.1007/s002940050160

Verdoes JC, Punt PJ, van der Berg P, Debets F, Stouthamer AH, van den Hondel CA (1994) Characterization of an efficient gene cloning strategy for Aspergillus niger based on an autonomously replicating plasmid: cloning of the nicB gene of A. niger. Gene 146:159-165. doi:10.1016/0378-1119(94)90288-7

Ward OP (2012) Production of recombinant proteins by filamentous fungi. Biotechnol Adv 30:1119-1139. doi:10.1016/j. biotechadv.2011.09.012

Wösten HA, van Veluw GJ, de Bekker C, Krijgsheld P (2013) Heterogeneity in the mycelium: implications for the use of fungi as cell factories. Biotechnol Lett 35:1155-1164 